\title{
Effects of electron-electron correlations on defect and interface states in amorphous $\mathrm{Si}$ and $\mathrm{SiO}_{2}$ systems
}

\author{
R. A. Barrio, ${ }^{*}$ R. J. Elliott, and A. S. Carrico ${ }^{\dagger}$ \\ Department of Theoretical Physics, Oxford University, 1 Keble Road, Oxford OX1 3NP United Kingdom
}

(Received 10 January 1986)

\begin{abstract}
The effects of electron-electron correlations are included in the nearest-neighbor tight-binding model of tetrahedrally coordinated covalent solids in the amorphous phase. The amorphous systems are represented by Bethe lattices and the electron-electron interactions are parametrized for a shortrange interaction in a Hubbard-like Hamiltonian. Self-consistent solutions are obtained in the Hartree-Fock approximation. If defects are included the self-consistent energy parameters vary with distance away from the defect but the solution is still possible if this variation falls away rapidly over a few shells. The cases of dangling bonds in $\mathrm{Si}$ and $\mathrm{SiO}_{2}$ are considered by terminating the Bethe lattice. The former shows localized states in the gap for two charge configurations, but $\mathrm{SiO}_{2}$ shows only one. The interface between $\mathrm{Si}$ and $\mathrm{SiO}_{2}$ is represented by joining two Bethe lattices. The self-consistent solution shows appreciable charge transfer on to the first $\mathrm{O}$ atom and consequential changes in the local density of states.
\end{abstract}

\section{INTRODUCTION}

The interface between crystalline $\mathrm{Si}$ and amorphous $\mathrm{SiO}_{2}$ has been extensively studied because of its practical importance in metal-oxide-semiconductor devices. Electronic traps localized at defects in the interface play an important role in the performance of such devices. Among the numerous theoretical treatments of the electronic problems, there have been several tight-binding models. ${ }^{1-3}$ All of these works simulate the amorphous substance by Bethe lattices and use a nearest-neighbor Hamiltonian with five parameters. In the paper by Laughlin et al. ${ }^{1}$ the Si crystal is also simulated by a Bethe lattice, and therefore the interface is represented by a single atom. It is interesting to note that most of the local properties of the interface are well treated in this way, and that the surface effects are confined to very few layers around the interface. Martinez and Yńduráin ${ }^{2}$ pointed out that, besides the two-dimensional nature of the interface (not taken into account by Ref. 1), the value of the site-energy parameter in atoms near the interface could influence the properties of the states near the Si gap. They also pointed out that the single-particle tight-binding model was particularly inadequate in this region since the strong local perturbation changes the many-body properties so that the local tight-binding parameters should be calculated self-consistently. In the preceding paper ${ }^{3}$ we have extended the calculation of a Si crystal with attached $\mathrm{SiO}_{2}$ Bethe lattices with particular reference to defect states at the interface, with various assumptions on the parameters involved.

On the other hand, Barrio et al. ${ }^{4}$ (BTMY) developed a theory for $a-\mathrm{Si}$ in which the two tight-binding parameters of the Weaire and Thorpe Hamiltonian ${ }^{5}$ can be calculated self-consistently by parametrizing the electron-electron correlations in a Hartree-Fock Hamiltonian. It is the purpose of this paper to apply the BTMY self-consistent method to the two-Bethe-lattice system of Ref. 1 in order to investigate the changes of the tight-binding parameters near the $\mathrm{Si}_{-} \mathrm{SiO}_{2}$ interface. In $\mathrm{Sec}$. II we describe the theory for a Si Bethe lattice; that is, we extend the calculation of BTMY to a complete five-parameter Hamiltonian. In Sec. III we show how the $\mathrm{SiO}_{2}$ Bethe lattice can be mapped into the preceding problem, and with these results we connect the two lattices in Sec. IV to study the interface. In Sec. V we summarize the important results of the work.

\section{SELF-CONSISTENT Si BETHE LATTICE}

If $|n \alpha\rangle$ represents one of the four $s p^{3}$ orbitals $\alpha$ centered on site $n$, the BTMY Hamiltonian can be written

$$
\begin{aligned}
H= & \sum_{n, \alpha, \beta} \Delta|n \alpha\rangle\left\langle n \beta\left|+\sum_{n, m, \alpha} V\right| n \alpha\right\rangle\langle m \alpha| \\
& +\frac{1}{2} U \sum_{n, \alpha} P_{\alpha \alpha}(n, n)|n \alpha\rangle\langle n \alpha| \\
& +K \sum_{n, m, \alpha} P_{\alpha \alpha}(n, m)|n \alpha\rangle\langle m \alpha|
\end{aligned}
$$

where the summations are over neighboring sites. The interaction terms $U$ and $K$ have been linearized and the density-matrix elements $P_{\alpha \beta}(n, n)$ are calculated selfconsistently using

$$
P_{\alpha \beta}(n, m)=\frac{-2}{\pi} \operatorname{Im} \int_{-\infty}^{E_{F}}\langle n \alpha|G| m \beta\rangle d E .
$$

The integral is over occupied states and the Green's function $G$ is found by solving the equations of motion for the Bethe lattice.

There are various ways by which the Hamiltonian (1) can be improved. First, it should be extended to include the electron spin $(\sigma)$, in order to account for spin effects in the electron-electron correlations. Second, intrasite correlations of the type

$$
J \sum_{n, \alpha, \beta} P_{\alpha \beta}^{\sigma}(n, n)|n \alpha, \sigma\rangle\langle n \beta, \sigma|
$$


are introduced. This was not done by BTMY because terms of this type break the symmetry around a defect site and the problem is then more complicated. Finally, instead of the Weaire-Thorpe Hamiltonian with $\Delta$ and $V$, all the possible interactions between $s$ and $p$ nearestneighbor orbitals are included. ${ }^{6}$ In this case one has $4 \times 4$ matrices for various $\alpha$ at each site in shell $n$ in the Bethe lattice. The site-energy matrix is defined as

$$
A_{1}^{\sigma}(n)=\left(\begin{array}{cccc}
U_{H}+\frac{1}{2} U P_{11}^{-\sigma}(n, n) & V_{1}+J P_{12}^{\sigma}(n, n) & V_{1}+J P_{13}^{\sigma}(n, n) & V_{1}+J P_{14}^{\sigma}(n, n) \\
V_{1}+J P_{21}^{\sigma}(n, n) & U_{H}+\frac{1}{2} U P_{22}^{-\sigma}(n, n) & V_{1}+J P_{23}^{\sigma}(n, n) & V_{1}+J P_{24}^{\sigma}(n, n) \\
V_{1}+J P_{31}^{\sigma}(n, n) & V_{1}+J P_{32}^{\sigma}(n, n) & U_{H}+\frac{1}{2} U P_{33}^{-\sigma}(n, n) & V_{1}+J P_{34}^{\sigma}(n, n) \\
V_{1}+J P_{41}^{\sigma}(n, n) & V_{1}+J P_{42}^{\sigma}(n, m) & V_{1}+J P_{34}^{\sigma}(n, n) & V_{H}+\frac{1}{2} U P_{44}^{-\sigma}(n, n)
\end{array}\right) .
$$

The subscript in the matrix notation states that the site in shell $n$ is connected to the previous shell by bond 1 . The interaction between sites connected by the same bond is

$$
W_{1}^{\sigma}(n)=\left(\begin{array}{cccc}
V_{2}+K P_{11}^{\sigma}(n, n+1) & V_{3} & V_{3} & V_{3} \\
V_{3} & V_{5} & V_{4} & V_{4} \\
V_{3} & V_{4} & V_{5} & V_{4} \\
V_{3} & V_{4} & V_{4} & V_{5}
\end{array}\right) .
$$

Of course, for a pure infinite lattice these matrices do not depend on the shell number $n$ and all the four directions are the same. However, if defects, like a dangling bond, or surfaces are introduced, this symmetry is broken and the values depend on shell and bond number.

We create a terminated Bethe lattice by removing bond 1 on site $n=1$. It is now necessary to solve an infinite chain of equations because all the quantities depend on $n$. It is reasonable to assume that beyond a certain shell $N$ this dependence is negligible and that locally the system behaves as a pure Bethe lattice. In practice, $N$ is chosen $a$ posteriori as the minimum number of shells that are needed in order to have variations smaller than certain desired number $\left(\sim 10^{-3}\right)$. Beyond shell $N$ the Green's function in the Bethe lattice obeys the equation of motion,

$$
\begin{aligned}
& G_{1}^{\sigma}(N, N+1)\left[E I-A_{1}^{\sigma}(N+1)\right] \\
& \quad=G_{1}^{\sigma}(N, N) W_{1}^{\sigma}(N)+\sum_{\alpha(\neq 1)} G_{\alpha}^{\sigma}(N, N+2) W_{\alpha}^{\sigma}(N) .
\end{aligned}
$$

Notice that we have assumed that $W_{1}^{\sigma}(N)=W_{1}^{\sigma}(N+1)$. Equation (6) can be solved by defining a transfer matrix

$$
G_{1}^{\sigma}(N, N+1)=G_{1}^{\sigma}(N, N) T_{1}^{\sigma}(N+1),
$$

and three matrices $S_{\alpha}$ which transform orbital $\alpha$ into orbital 1; therefore,

$$
\begin{aligned}
G_{\alpha}^{\sigma}(N, N+2) & =S_{\alpha}^{-1} G_{1}^{\sigma}(N, N+2) S_{\alpha} \\
& =G_{1}^{\sigma}(N, N+1) S_{\alpha}^{-1} T_{1}^{\sigma}(N+2) S_{\alpha} .
\end{aligned}
$$

Since $T_{1}^{\sigma}(N+2)=T_{1}^{\sigma}(N+1)$, substitution of (7) and (8) into (6) gives a solution for $T_{1}^{\sigma}(N+1)$. Then one obtains a chain of equations for the rest of the $T$ matrices,

$$
\begin{aligned}
T_{1}^{\sigma}(n)=W_{1}(n-1) & {\left[E I-A_{1}^{\sigma}(n)\right.} \\
& \left.-\sum_{\alpha(\neq 1)} S_{\alpha}^{-1} T_{1}^{\sigma}(n+1) W_{1}^{\sigma}(n) S_{\alpha}\right)^{-1},
\end{aligned}
$$

for $n=2, \ldots, N$. With these matrices all Green's functions needed can be built sequentially, starting with

$$
\begin{aligned}
G_{1}^{\sigma}(1,1)= & {\left[E I-A_{1}^{\sigma}(1)-\sum_{\alpha(\neq 1)} S_{\alpha}^{-1} T_{1}^{\sigma}(2) W_{1}^{\sigma}(1) S_{\alpha}\right.} \\
& \left.-\left[T_{1}^{\sigma}(2) W_{1}^{\sigma}(2)\right]\right]^{-1} .
\end{aligned}
$$

The term in square brackets is present in the pure lattice, but is absent for a dangling-bond defect in orbital 1.

We can work out all the other correlations needed using the rotations $S_{\alpha}$ and equations like (8); for instance, the correlation between sites 1 and 2 , connected by bond $\alpha$, in the representation where the first row is $\alpha=1$, is

$$
\begin{aligned}
G_{1}^{\sigma}(1,2) & =S_{\alpha} G_{\alpha}^{\sigma}(1,2) S_{\alpha}^{-1} \\
& =S_{\alpha} G_{1}^{\sigma}(1,1) T_{\alpha}^{\sigma}(2) S_{\alpha}^{-1} \\
& =S_{\alpha} G_{1}^{\sigma}(1,1) S_{\alpha}^{-1} T_{1}^{\sigma}(2)
\end{aligned}
$$

Using the equations for site 2 , we find

$$
G_{1}^{\sigma}(2,2)=\left\{\left[W_{1}^{\sigma}(1)\right]^{-1}+G_{1}^{\sigma}(1,2)\right\}^{-1} T_{1}^{\sigma}(2) .
$$

These relations can be extended to further shells. The local densities on each shell are

$$
\rho^{\sigma}(n)=\frac{-1}{\pi} \operatorname{Im} \operatorname{Tr} G_{1}^{\sigma}(n, n),
$$

TABLE I. Parameters used for the Si Bethe lattice (in eV). The parameters in this table differs from those given in Table I of Ref. 3 in terms of the use of a Bethe lattice instead of a crystal, and because of a shift in the energy origin of $5.36 \mathrm{eV}$.

\begin{tabular}{cccccccccc}
\hline \hline$U_{H}$ & $V_{1}$ & $V_{2}$ & $V_{3}$ & $V_{4}$ & $V_{5}$ & $U$ & $J$ & $K$ \\
\hline 2.7 & -1.9 & -5.44 & -0.2 & -0.4 & 0.6 & 3 & 1 & 1 \\
\hline \hline
\end{tabular}


TABLE II. Site-energy matrix elements (in eV) and charge densities for a Si Bethe lattice with a dangling bond as a function of the shell-generation number $n$ (blanks mean that the value is the bulk one, which is given at the bottom of each column). The parameters in this table differs from those given in Table I of Ref. 3 in terms of the use of a Bethe lattice instead of a crystal, and because of a shift in the energy origin of $5.36 \mathrm{eV}$.

\begin{tabular}{|c|c|c|c|c|c|c|c|}
\hline \multirow[b]{2}{*}{$n$} & \multirow[b]{2}{*}{ Spin } & \multirow[b]{2}{*}{$A_{11}$} & \multirow[b]{2}{*}{$A_{22}$} & \multirow[b]{2}{*}{$A_{12}$} & \multirow[b]{2}{*}{$A_{24}$} & \multicolumn{2}{|c|}{ Charge density } \\
\hline & & & & & & $P_{11}$ & $P_{22}$ \\
\hline \multirow[t]{2}{*}{1} & $\uparrow$ & 3.28 & 4.18 & -1.74 & -1.82 & 0.88 & 0.53 \\
\hline & $\downarrow$ & 5.35 & 4.18 & -1.81 & -1.82 & 0.16 & 0.47 \\
\hline \multirow[t]{2}{*}{2} & $\uparrow$ & 4.14 & 4.21 & -1.81 & & 0.53 & 0.51 \\
\hline & $\downarrow$ & 4.30 & 4.20 & -1.81 & & 0.47 & 0.49 \\
\hline \multirow[t]{2}{*}{3} & $\uparrow$ & 4.15 & & -1.82 & & & \\
\hline & $\downarrow$ & 4.25 & & -1.81 & & & \\
\hline \multirow[t]{2}{*}{4} & $\uparrow$ & 4.20 & & -1.82 & & & \\
\hline & $\downarrow$ & 4.22 & & -1.82 & & & \\
\hline \multirow[t]{2}{*}{5} & $\uparrow$ & 4.20 & & & & 0.50 & 0.50 \\
\hline & $\downarrow$ & 4.20 & & & & 0.50 & 0.50 \\
\hline$\infty$ & $\uparrow \downarrow$ & 4.2 & 4.2 & -1.82 & -1.82 & 0.5 & \\
\hline
\end{tabular}

and the various elements of the density matrix are

$$
P_{\alpha \beta}^{\sigma}(n, m)=\frac{-2}{\pi} \operatorname{Im}\left(\int_{-\infty}^{E_{F}} G_{\alpha \beta}^{\sigma}(n, m) d E\right) .
$$

This integral is done in the complex plane following the method described by Robbins and Falicov ${ }^{7}$ in order to minimize the computing time, and the Fermi level is found according to the sum rule

$$
\frac{1}{N} \sum_{n=1}^{N} \sum_{\alpha=1}^{4}\left[P_{\alpha \alpha}^{\sigma}(n, n)+P_{\alpha \alpha}^{-\sigma}(n, n)\right]=4
$$

The self-consistency is obtained by feeding back into (4) and (5) the numbers calculated with (14). The values of the parameters $U, J$, and $K$ are chosen to reproduce the bands for the pure case [Eq. (10) with the term in square brackets in it]. For instance, the combined value of $U_{H}+\frac{1}{2} U P_{11}^{-\sigma}(n, n)$ has to be the same as $U_{H}$ for the normal tight-binding Hamiltonians.

The dangling bond is then studied by removing the term in square brackets in (10) and the occupancy of the orbital is stated by the initial values of $P_{11}^{\dagger}(1,1)$ and $P_{11}^{\downarrow}(1,1)$ to give electron numbers 0,1 , or 2 in Eq. (15).

In Table II we summarize the results, obtained with the parameters shown in Table I, for a dangling bond in $n=1$ with initially one electron with $\sigma=\downarrow$. We give only the values of the different matrix elements of $A$ because the variation of $W_{11}$ with $n$, from the bulk value of $5 \mathrm{eV}$, is negligible $\left(<10^{-2}\right)$. Here, as in the BTMY theory, the value of $U$ that reproduces the bands is $3 \mathrm{eV}$, much smaller than the typical atomic values of the order of 10-12 $\mathrm{eV}$. This is attributed to the fact that polarization terms due to electron rearrangement around a defect in a solid can be absorbed into $U$ in a Hartree-Fock treatment. If polarization terms $(\sim D)$ are included, then the effective $U$ is $U_{\text {eff }}=U-2 D$, and $D$ may be as large as $5-6 \mathrm{eV}$ in some insulators, although in $\mathrm{Si}$ it is probably $3 \mathrm{eV} .^{8}$

We observe that $N$ in this calculation is 5 ; that is, that the effects of the dangling bond are localized around the defect site. The largest variation is seen in $A_{11}$; changes are as large as $27 \%$.

We notice that the change-density fluctuations around the dangling bond are localized in agreement with the results of BTMY. A simple calculation using the HaldaneAnderson model for a dangling bond in Si gives similar charge densities around the defect. ${ }^{8}$

In Fig. 1 we show the local densities of states from the final results of Table II; we notice two dangling-bond states in the gap corresponding to the singly occupied and doubly occupied defects, separated by $0.7 \mathrm{eV}$. It is worth remarking that the self-consistency over all the lattice is necessary for this reduction; if electron-electron correlations were permitted only on the defect site, this difference would have been of the order of $U=3 \mathrm{eV}$.

Our results agree with the BTMY theory because we show here that the most important term in the Hamiltonian is $U$. The quantities are somewhat different but the qualitative behavior is consistent with BTMY.

\section{III. $\mathrm{SiO}_{2}$ BETHE LATTICE}

The $\mathrm{SiO}_{2}$ lattice can be treated in the same way since the $\mathrm{Si}$ sites form a lattice topologically analogous to the $\mathrm{Si}$ one, except that there are oxygen atoms between the $\mathrm{Si}$ sites. The Si-O interactions are shown in Fig. 2, where we have neglected oxygen $p p \pi$ interactions, for two reasons: (1) With these interactions the equations of motion de- 


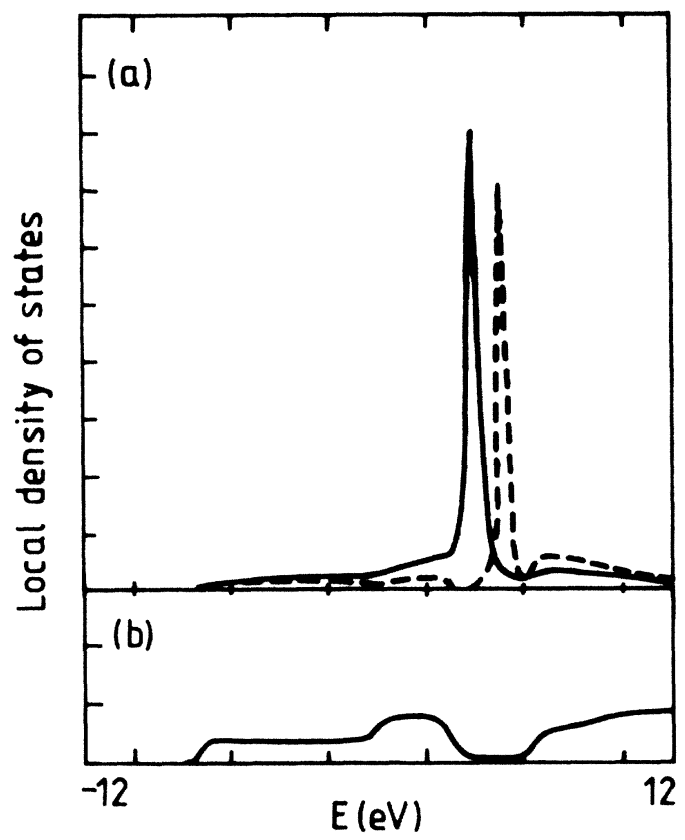

FIG. 1. (a) Local density of states at the dangling bond terminating a Si Bethe lattice. Solid line for spin orbital $\sigma=\downarrow$. Dashed line for spin orbital $\sigma=\uparrow$ (double occupancy). The calculation is made using the parameters in Table $I$ and an imaginary part to the energy $\sim 10^{-1} \mathrm{eV}$. (b) Density of states in the bulk.

pend on the dihedral angles which therefore need to be specified for every bond, and (2) these interactions have very little effect on the results near the Si gap. ${ }^{2}$ Using the equation of motion one transforms the oxygen coordinates away by renormalizing the $\mathrm{Si}-\mathrm{Si}$ bonds, which means that the equations are the same as (6), except that the effective interaction matrices $A^{\text {eff }}$ and $W^{\text {eff }}$ contain the oxygen parameters and depend on the energy. We define

$$
\begin{aligned}
& A_{1, \sigma}^{\text {eff }}(n)=A_{1}^{\sigma}(n)+\Sigma(E), \\
& W_{1, \sigma}^{\text {eff }}(n)=W_{1}^{\sigma}(n)+C(E),
\end{aligned}
$$

where we have separated the terms that depend on the en-

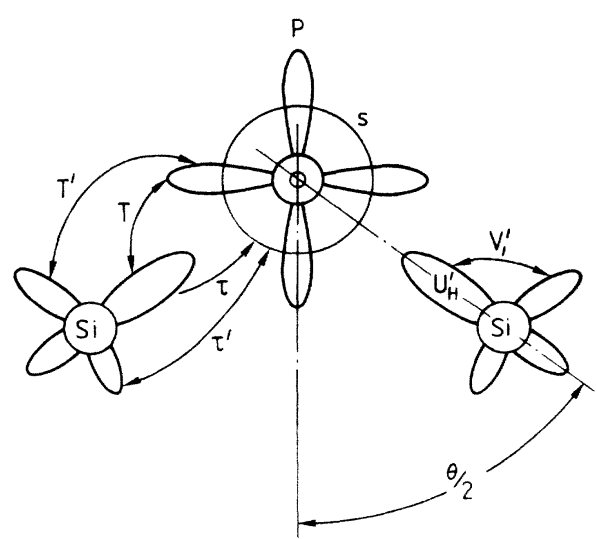

FIG. 2. Tight-binding parameters used to construct a $\mathrm{SiO}_{2}$ Bethe lattice. ergy. The first terms on the right-hand side of (16) are the matrices in the form (4) and (5) with different values of $U_{H}, V_{1}, U, J$, and $K$, while $V_{i}=0$ for $i=2,3,4,5$. Explicitly, the energy-dependent terms are

$$
\begin{aligned}
& \Sigma_{\alpha \alpha}(E)=\frac{T^{2}+3\left(T^{\prime}\right)^{2}}{E-E_{p}}+\frac{\tau^{2}+3\left(\tau^{\prime}\right)^{2}}{E-E_{s}}, \\
& \Sigma_{\alpha \beta}(E)=\frac{2\left[T T^{\prime}+\left(T^{\prime}\right)^{2}\right]}{E-E_{p}}+\frac{2\left[\tau \tau^{\prime}+\left(\tau^{\prime}\right)^{2}\right]}{E-E_{s}}, \alpha \neq \beta \\
& C_{11}(E)=\frac{T^{2} \cos \theta}{E-E_{p}}+\frac{\tau^{2}}{E-E_{s}}, \\
& C_{1 \alpha}(E)=\frac{T T^{\prime} \cos \theta}{E-E_{p}}+\frac{\tau \tau^{\prime}}{E-E_{s}}=C_{\alpha 1}, \quad \alpha=2,3,4 \\
& C_{\alpha \beta}(E)=\frac{\left(T^{\prime}\right)^{2} \cos \theta}{E-E_{p}}+\frac{\left(\tau^{\prime}\right)^{2}}{E-E_{s}}, \quad \alpha, \beta=2,3,4 .
\end{aligned}
$$

The values $E_{p}$ and $E_{s}$ are eigenvalues of the oxygen $2 s$ and $3 p$ orbitals, respectively.

The pure Bethe lattice can be solved using the operations in Sec. II. Using the parameters listed in Table III, the results are shown in Fig. 3(b). The Bethe-lattice density of states stretches over a broad energy range with a gap between 0 and $8 \mathrm{eV}$.

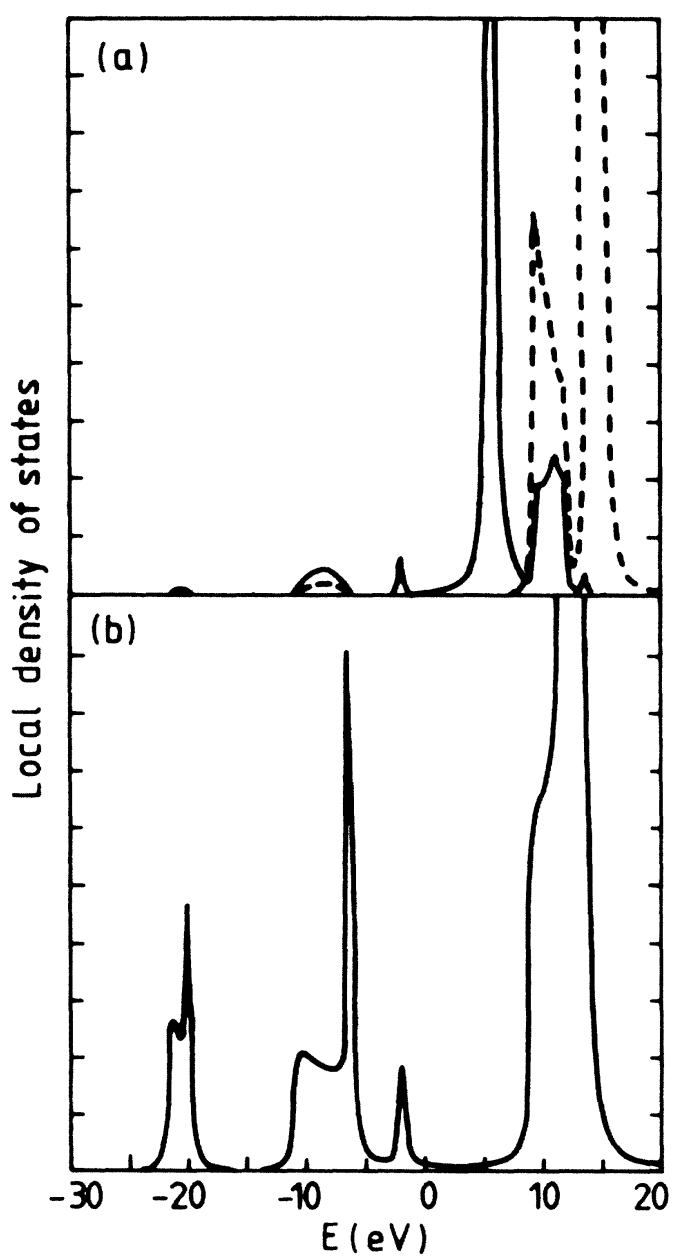

FIG. 3. Same as Fig. 1 for a $\mathrm{SiO}_{2}$ Bethe lattice with parameters in Table III. 
TABLE III. Parameters used for the $\mathrm{SiO}_{2}$ Bethe lattice (energies in eV). The parameters in this table differs from those in Table II of Ref. 3 because of a shift in origin of $5.36 \mathrm{eV}$ (cf. $E_{s}, E_{p}$, and $A_{11}$; the latter should be compared to $\left.U_{H 0}\right)$.

\begin{tabular}{cccccccccccc}
\hline \hline$U^{1}$ & $V_{1}^{1}$ & $E_{p}$ & $E_{s}$ & $T$ & $T^{1}$ & $\tau$ & $\tau^{1}$ & $(\mathrm{deg})$ & $U$ & $J$ & $K$ \\
\hline 5.6 & -1.52 & -0.3 & -15.5 & -7.0 & -0.65 & -7.2 & -0.2 & 144 & 4 & 2 & 2 \\
\hline \hline
\end{tabular}

The dangling-bond case can be treated as before, allowing for a modification of the energy-dependent terms $\Sigma$ on the defect site. For a dangling bond on site $n=1$ and orbital 1 , there are only three adjacent oxygens, and

$$
\begin{aligned}
& \Sigma_{11}(E)=\frac{3\left(T^{\prime}\right)^{2}}{E-E_{p}}+\frac{3\left(\tau^{\prime}\right)^{2}}{E-E_{s}}, \\
& \Sigma_{1 \alpha}(E)=\frac{T T^{\prime}+2\left(T^{\prime}\right)^{2}}{E-E_{p}}+\frac{\tau \tau^{\prime}+2\left(\tau^{\prime}\right)^{2}}{E-E_{s}}=\Sigma_{\alpha 1}(E), \\
& \Sigma_{\alpha \alpha}(E)=\frac{T^{2}+2\left(T^{\prime}\right)^{2}}{E-E_{p}}+\frac{\tau^{2}+2\left(\tau^{\prime}\right)^{2}}{E-E_{s}}, \\
& \Sigma_{\alpha \beta}(E)=\frac{2 T T^{\prime}+\left(T^{\prime}\right)^{2}}{E-E_{p}}+\frac{2 \tau \tau^{\prime}+\left(\tau^{\prime}\right)^{2}}{E-E_{s}}, \quad \alpha, \beta \neq 1 .
\end{aligned}
$$

The results of the calculation for the dangling bond are summarized in Table IV. One notices immediately that the effect of electron correlations in the $\mathrm{SiO}_{2}$ lattice is practically concentrated on the first shell around the defect. The density of states for this system is shown in Fig. 3(a). The single dangling-bond state produces a sharp level in that gap (solid line), while the second orbital produces resonances in the conduction bands (dashed line).

\section{INTERFACE}

There are various ways in which an interface between the two Bethe lattices can be built $;^{1}$ of these we choose the one in which the last atom in the Si Bethe lattice is surrounded by three $\mathrm{Si}$ atoms and one oxygen atom, and the first atom in the $\mathrm{SiO}_{2}$ is surrounded by four oxygen atoms, just as the rest in the $\mathrm{SiO}_{2}$ lattices. This models the interface between $\mathrm{Si}(111)$ and $a-\mathrm{SiO}_{2}$, which has been studied by us $^{3}$ without the self-consistent theory. The notation for the layers is illustrated in Fig. 4. Then the equations of motion near the interface are

$$
\begin{aligned}
& G_{1}^{\sigma}(-1,1)\left[E I-A_{1}^{\sigma}(1)-\sum_{\alpha(\neq 1)} S_{\alpha}^{-1} T_{1}^{\sigma}(2) W_{1}^{\sigma}(1) S_{\alpha}\right)=G_{1}(-1,-1) W_{1}(0) \\
& G_{1}^{\sigma}(-1,-1)\left[E I-A_{1, \sigma}^{\mathrm{eff}}(1)-\sum_{\alpha(\neq 1)} S_{\alpha}^{-1} T_{1, \sigma}^{\mathrm{eff}}(-2) W_{1, \sigma}^{\mathrm{eff}}(-1) S_{\alpha}\right)=I+G_{1}^{\sigma}(-1,1) W_{-1, \sigma}^{\mathrm{eff}}(0) .
\end{aligned}
$$

The site-energy matrix $A_{1}^{\sigma}(1)$ now depends on the energy through the oxygen bond; therefore, to (4), one has to add the terms

$$
\Sigma_{11}(E)=\frac{T^{2}}{E-E_{p}}+\frac{\tau^{2}}{E-E_{s}},
$$

$$
\begin{aligned}
& \Sigma_{\alpha \beta}(E)=\frac{\left(T^{\prime}\right)^{2}}{E-E_{p}}+\frac{\left(\tau^{\prime}\right)^{2}}{E-E_{s}} \\
& \Sigma_{1 \alpha}(E)=\frac{T T^{\prime}}{E-E_{p}}+\frac{\tau \tau^{\prime}}{E-E_{s}}=\Sigma_{\alpha 1}(E), \quad \alpha, \beta \neq 1 .
\end{aligned}
$$

TABLE IV. Site-energy matrix elements (in eV) and charge densities for a $\mathrm{SiO}_{2}$ Bethe lattice with a dangling bond at shell $n=1$ (blank entries indicate bulk values given in last row). The parameters in this table differs from those in Table II of Ref. 3 because of a shift in origin of $5.36 \mathrm{eV}$ (cf. $E_{s}, E_{p}$, and $A_{11}$; the latter should be compared to $U_{H 0}$ ).

\begin{tabular}{cccccccc}
\hline \hline$n$ & Spin & $A_{11}$ & $A_{22}$ & $A_{12}$ & $A_{34}$ & $P_{11}$ Charge density & $P_{22}$ \\
\hline 1 & $\uparrow$ & 5.81 & 7.54 & -1.32 & -1.57 & 0.06 & 0.43 \\
1 & $\downarrow$ & 13.98 & 7.56 & -1.62 & -1.58 & 0.94 & 0.56 \\
2 & $\uparrow$ & 7.52 & 7.53 & -1.58 & & 0.44 & 0.48 \\
2 & $\downarrow$ & 7.56 & 7.53 & -1.58 & & 0.55 & 0.52 \\
3 & $\uparrow$ & 7.53 & & & & & \\
3 & $\downarrow$ & 7.53 & & & & & \\
4 & $\uparrow$ & & & & & & \\
4 & $\downarrow$ & & & & & & \\
$\infty$ & $\uparrow \downarrow$ & 7.53 & 7.53 & -1.58 & -1.58 & 0.50 & 0.50 \\
\hline \hline
\end{tabular}




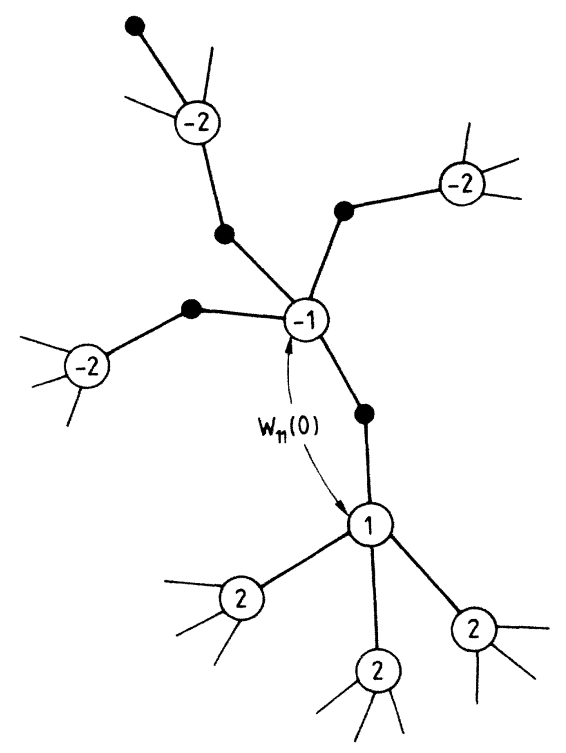

FIG. 4. $\mathrm{Si}-\mathrm{SiO}_{2}$ interface showing the shell-generation numbers $n$ for each Si atom on both sides of the interface.

The interactions through the bond at the interface $n=0$ are straightforward extensions of $W^{\text {eff }}$,

$$
W^{\sigma}(0)=W_{1 \sigma}^{\text {eff }}(0)=W_{1 \sigma}^{\text {eff }}(2),
$$

except for the first diagonal term, which is

$$
\left\langle 1\left|W_{1}^{\sigma}(0)\right| 1\right\rangle=K^{1} P_{11}^{\sigma}(1,-1)
$$

and

$$
\left\langle 1\left|W_{1 \sigma}^{\mathrm{eff}}(0)\right| 1\right\rangle=K^{1} P_{11}^{\sigma}(-1,1),
$$

where $K^{1}$ is the value in the $\mathrm{SiO}_{2}$ lattice.

The results, using the same parameters as in the preceding sections, are shown in Table V. One immediately notices that the $\mathrm{SiO}_{2}$ lattice is not perturbed much by the presence of the interface, but the Si lattice is strongly affected in the first shell. There is a charge defect in the interface; the charge-density difference between the first two shells on the Si side is $\sim 0.2$ electrons. This charge has been sucked by the oxygen bond in the interface, resulting in a decrease in the site energy $U_{H}$ of the $\mathrm{Si}$ with three $\mathrm{Si}$ bonds. Figure 5 (b) gives the local density of states on $\mathrm{Si}$ atoms well away from the interface in the $\mathrm{Si}$ (solid line) and $\mathrm{SiO}_{2}$ (dashed line). The results are similar to those within the single Bethe lattices of each species [cf. Figs. $1(b)$ and 3(b)]. Figure 5(a) gives the same result for $\mathrm{Si}$ atoms next to the interface. There is little change on the $\mathrm{SiO}_{2}$ side, but the $\mathrm{Si}$ attached to the other lattice is strongly affected.

\section{CONCLUSIONS}

We have extended the BTMY theory in order to include all the nearest-neighbor interactions between $s p^{3}$ states in $\mathrm{Si}$. The results for the pure-Si lattice and for the dangling bond are in qualitative agreement with the results of BTMY. We believe that the present treatment is more realistic since we use a better tight-binding Hamiltonian.
TABLE V. Site-energy matrix elements (in $\mathrm{eV}$ ) for Si sites in the $\mathrm{Si}_{-} \mathrm{SiO}_{2}$ interface shown in Fig. 4 (both spin values give the same result).

\begin{tabular}{cllll}
\hline \hline$n$ & $A_{11}$ & $A_{22}$ & $A_{12}$ & $A_{34}$ \\
\hline$-\infty$ & 7.53 & 7.53 & -1.58 & -1.58 \\
-2 & 7.505 & 7.505 & & \\
-1 & 7.503 & 7.506 & -1.59 & -1.59 \\
1 & 3.61 & 4.25 & -1.83 & -1.81 \\
2 & 4.18 & 4.21 & & \\
3 & 4.19 & 4.20 & & -1.82 \\
$\infty$ & 4.2 & 4.2 & -1.82 & \\
\hline \hline
\end{tabular}

We find two dangling-bond states in the gap that correspond to different occupancy states; the separation between the two is $\sim 0.7 \mathrm{eV}$, in agreement with that measured in spin-resonance experiments. ${ }^{9}$ This is a consequence of the self-consistency performed in the whole lattice and stresses the importance of screening effects in a

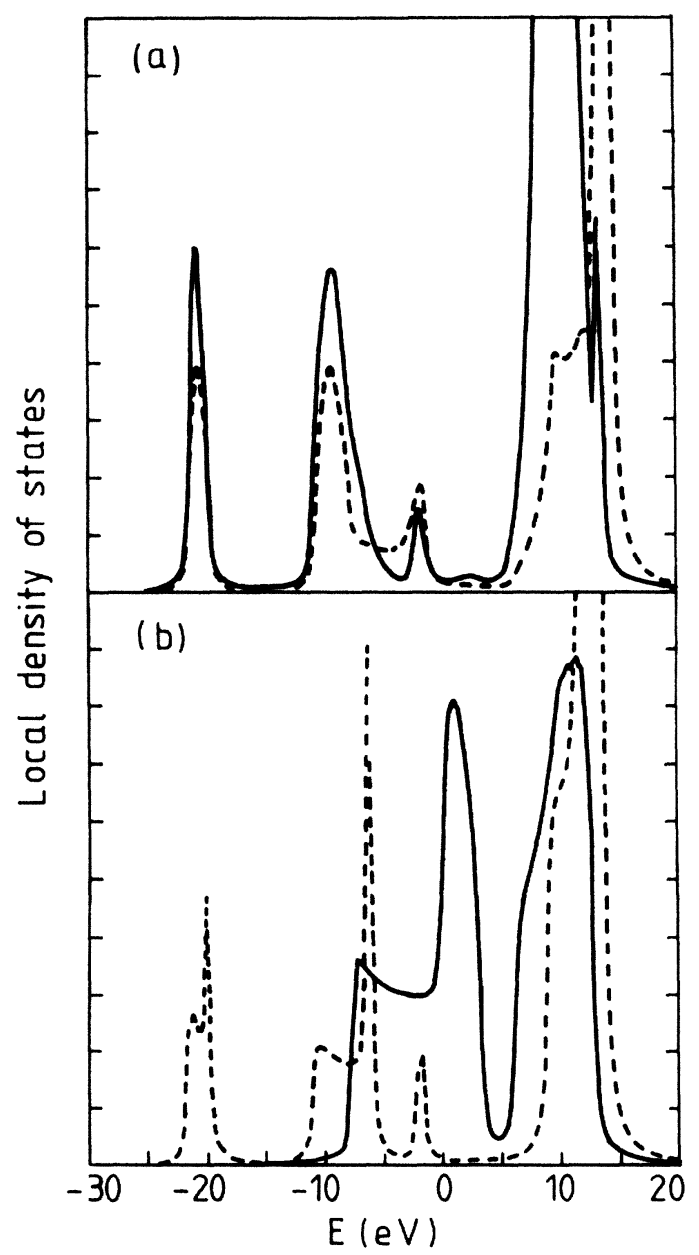

FIG. 5. (a) Local densities on the Si orbitals next to the Si$\mathrm{SiO}_{2}$ interface on the $\mathrm{Si}$ side (-) and the $\mathrm{SiO}_{2}$ side $(---)$. (b) Local densities on $\mathrm{Si}$ atoms in shells far away from the interface on the $\mathrm{Si}$ side (_- ) and the $\mathrm{SiO}_{2}$ side $(---)$. 
lattice. In accordance with BTMY we found a net magnetic moment localized in the dangling bond, $P_{11}^{\dagger}(1)-P_{11}^{\downarrow}(1)=0.72$ electrons, as expected. ${ }^{10}$

In the $\mathrm{SiO}_{2}$ lattice the effects of the dangling bond are much more localized, there are no multiple occupancy states in the gap, the singly occupied state is found at $\sim 5.75 \mathrm{eV}$, and the doubly occupied state is as resonance in the conduction band at $\sim 9.5 \mathrm{eV}$. The difference in energy of $3.75 \mathrm{eV}$ is practically the value of $U=4 \mathrm{eV}$, reflecting the fact that the screening of the electrons in the lattice is much less severe in this case.

The results for the interface show an unexpected effect, since there is charge imbalance there resulting in a change in the wrong direction of the effective $U_{H}$ for the Si with one oxygen bond. In previous calculations ${ }^{1,2}$ this value was taken as $\frac{1}{4}\left(3 U_{H}+U_{H}^{1}\right)=5.3 \mathrm{eV}$, while the value calculated here was $3.61 \mathrm{eV}$, a large discrepancy. This is at- tributed to the ionicity of the oxygen-silicon bond that attracts the electron in the interface bond toward the oxygen. It will be necessary to construct other interfaces with two and three oxygen bonds in order to investigate the variation of $U_{H}$ with the number of oxygen bonds. It will also be necessary to perform a self-consistent calculation of the type described here to the crystalline interfaces of reference, ${ }^{3}$ in order to treat the $P_{b}$ center in the interface. Such a calculation is in progress.

\section{ACKNOWLEDGMENTS}

We are grateful to Dr. J. Taguena-Martinez and Professor Beall Fowler for useful discussions. This work was supported in part by the U.S. Army through its European Research Office. One of us (R.A.B.) wishes to thank Oxford University for its hospitality.
*Permanent address: Instituto de Investigaciones en Materiales, Universidad Nacional Autónoma de México, Apdo. Postal 70-360, 04510 Mexico D.F., Mexico.

†Permanent address: DFTE-Centro de Ciências Exatas, UFRN, 59000 Natal-NR, Brazil.

${ }^{1}$ R. B. Laughlin, J. D. Joannopoulos, and D. J. Chadi, Phys. Rev. B 21, 5733 (1980).

${ }^{2}$ E. Martinez and F. Yńduráin, Phys. Rev. B 25, 6511 (1982).

${ }^{3}$ A. S. Carrico, R. J. Elliott, and R. A. Barrio preceding paper, Phys. Rev. B 34, 872 (1986).

${ }^{4}$ R. A. Barrio, J. Taguena-Martinez, E. Martinez, and F.
Yńduráin, J. Non-Cryst. Solids 72, 181 (1985).

${ }^{5}$ D. Weaire and M. F. Thorpe, Phys. Rev. B 4, 2508 (1971).

${ }^{6}$ J. C. Slater, Quantum Theory of Molecules and Solids (McGraw-Hill, New York, 1965).

${ }^{7}$ M. O. Robbins and L. M. Falicov, Phys. Rev. B 29, 1333 (1984).

${ }^{8}$ W. B. Fowler and R. J. Elliott (private communication).

${ }^{9}$ E. H. Poindexter and P. J. Caplan, Prog. Surf. Sci. 14, 201 (1983).

${ }^{10}$ See, for instance, Amorphous Semiconductors, edited by M. H. Brodsky (Springer-Verlag, Berlin, 1979). 